\title{
Statin use and hip fractures in U.S. kidney transplant recipients
}

\author{
Chandan Vangala', Colin R. Lenihan ${ }^{2 *}$, Maria E. Montez-Rath ${ }^{3}$, Sumi Sukumaran Nair ${ }^{4}$, Sankar D. Navaneethan ${ }^{5}$, \\ Venkat Ramanathan ${ }^{1,6}$ and Wolfgang C. Winkelmayer ${ }^{7}$
}

\begin{abstract}
Background: Basic and translational research supports beneficial effects of statins on bone metabolism. Clinical studies suggest that statin use may reduce the risk of hip fractures in the general population. Whether statin use is associated with hip fracture risk in kidney transplant recipients, a particularly high-risk group for this outcome, is unknown.

Methods: From the U.S. Renal Data System (2007-2011), we identified all hip fracture events recorded in Medicare billing claims of first-time kidney transplant recipients. We then matched all cases to an unlimited number of controls on age ( \pm 3 years), sex, race (black vs. non-black), and time since transplant. Cases and controls were required to have $>1$ year of Medicare Parts $A+B+D$ coverage and be without a recorded history of hip fracture. We ascertained any statin use in the previous year and defined adherent statin use as those who had filled prescriptions for statins to cover $>80 \%$ of days in that year (proportion of days covered, PDC). We ascertained several potential confounders (demographics, comorbidities, BMI, transplant-related factors) and applied conditional logistic regression with multiple imputation for missing data to estimate odds ratios (OR) and $95 \%$ confidence intervals $(\mathrm{Cl})$.

Results: We identified 231 hip fracture cases (mean age 51.8 years; $53 \%$ female; $11.3 \%$ black; 6.9 years from transplant, and 9.9 years from ESRD) and 15,575 matched controls. Any prior statin use was present in $64.1 \%$ of cases and $60.3 \%$ of controls with $37.2 \%$ of cases and $33.9 \%$ of controls being found adherent. Unadjusted conditional logistic regression showed an OR of 1.17 (0.89-1.54) for any statin use, and a fully-adjusted OR of 0.89 (0.67-1.19). Compared with statin non-users, the adjusted OR for patients with lesser adherence (PDC $\leq 80 \%)$ and those with greater adherence (PDC >80\%) were 0.93 (0.66-1.31) and 0.87 (0.63-1.20), respectively.
\end{abstract}

Conclusion: Statin use was not associated with hip fracture risk in first-time kidney transplant recipients.

Keywords: End-stage renal disease, Hip fracture, Outcomes, Drug safety, Case-control, USRDS

\section{Background}

Hip fractures are devastating events, conferring morbidity, mortality, disability, and high costs to the health care system. In 2005, the general population's age-adjusted incidence rates were 369 and 794 per 100,000 personyears in men and women, respectively [1]. When compared with the general population, patients on dialysis have been shown to carry a more than 4 -fold risk of hip fracture [2]. However, kidney transplant recipients

\footnotetext{
*Correspondence: clenihan@stanford.edu

${ }^{2}$ Division of Nephrology, Stanford University School of Medicine, 777 Welch

Road Suite DE, Palo Alto, CA 94304, USA

Full list of author information is available at the end of the article
}

(KTRs) represent a select group of patients that have also maintained a highly elevated hip fracture risk, carrying an even 1.34-fold higher risk than patients on dialysis [3]. The mineral bone disease associated with chronic kidney disease prior to transplant, immunosuppressive regimens utilizing corticosteroids, and age-related osteoporosis are mechanisms that interact to distort bone health. The risk for hip fracture is greatest in the first years post-transplant, declining to equal that of patients on dialysis by the end of the second post-transplant year [3]. Given the increased mortality, significant morbidity, and large healthcare costs associated with hip fracture, 
any potentially modifiable risk factors including medications should be carefully examined.

Basic and translational studies have identified multiple potential mechanisms by which the 2-hydroxy-3-methylglutaryl coenzyme A reductase inhibitors (statins) affect bone metabolism $[4,5]$. Furthermore, statins have been associated with a reduced risk of hip fracture in the 1) elderly general, 2) mostly male Veterans Affairs, and 3) post-menopausal female populations [6-8].

Considering the elevated risk of cardiovascular disease associated with kidney disease and concurrent immunosuppressive medications, statins are widely prescribed for KTRs. Indeed, current guidelines recommend statin treatment for all adult KTRs (leaving some room for discretion in patients aged less than 30 years with fewer cardiovascular risk factors) [9]. A large, retrospective, multicenter international study of kidney transplant outcomes, the Patient Outcomes in Renal Transplantation study, and a separate, prospective observational study at seven transplant centers in the US and Canada estimated that $\sim 40 \%$ of patients in the 6 months post-transplant were prescribed a statin $[10,11]$. While statins are utilized to diminish this cardiovascular risk in KTRs, the sum of the evidence supporting statin use is not fully conclusive [12]. Therefore, the possibility of added benefit from bone fracture prevention would further justify statin exposure in KTRs. The incidence of hip fractures in first time KTRs has decreased from 1997 to 2010 possibly due to a number of plausible explanations, including adoption of steroid sparing or minimizing protocols, transition to tacrolimus, bisphosphonate use, and cinacalcet use [13]. During the same time, statin use in KTR has also likely increased and may have contributed towards this positive trend.

To our knowledge, there is no data to support an association between statin use and hip fracture risk in the kidney transplant population. We, therefore, conducted the present study to challenge the null hypothesis of no association between statin use and post-transplant hip fracture in U.S. KTRs.

\section{Methods}

\section{Study design and source population}

We conducted a retrospective, nested, matched case control investigation of all first-time KTRs recorded in the United States Renal Data System (USRDS). The USRDS is a national registry that contains detailed information about KTRs via the United Network of Organ Sharing (UNOS) and Medicare claims [14]. First time KTRs who maintained a functioning transplant at any point in time between January 1, 2007 to December 31, 2011 and who had Medicare as their primary health care payer were considered eligible for study. Since diagnoses and prescription information came from Medicare claims, all cases and controls were required to have Medicare Parts A, B, D and be registered for the lowincome subsidy for at least 1 year prior to index date.

The date of hip fracture diagnosis was defined as the index date. We identified hip fracture events using the inpatient International Classification of Diseases, 9th Revision (ICD-9) diagnosis codes 820.xx or 821.xx. Additionally, hip fracture cases required any ICD-9 surgical procedure code among 78.55, 79.15, 79.35, 81.52, 81.51, $79.05,79.25$, or 81.40 within 7 days of hip fracture diagnosis [13]. For each case, all available and eligible controls were matched at the index date on transplant vintage (year of transplant), age (within 3 years), sex, and race (non-African American vs. African American). We employed this conditional risk-set matching scheme to maintain parsimony in our outcomes models while controlling for the potential confounding of these previously established risk factors. Parsimony was deemed important to avoid model over-fitting as we had to expect that the number of cases would be small relatively to the number of variables to be considered. Patients with any prior organ transplant or previous evidence of hip fracture were excluded from the study, and controls could subsequently become cases (see Additional file 1: Figure S1).

\section{Exposure of interest}

We focused on statin use as the differentiating exposure. Medicare Part D prescription claims were examined to identify use of any statin and patients' degree of adherence to these medications. Prior to the index date, any use was defined as at least 1 prescription claim within the last 365 days. Among any statin users, we labeled lesser use among those KTRs who received pharmacydispensed pills covering less than $80 \%$ of the 365 days prior to the index date. Lastly, higher use was noted as having statin pills dispensed that covered $80 \%$ or more of the year prior to the index date.

\section{Covariates}

Patient characteristics were abstracted from the patient and transplant files in the USRDS and comorbidities were ascertained from Medicare claims preceding the index date by $\geq 1$ year (see Additional file 1: Table S1 for specifications). Comorbidities such as diabetes mellitus, cardiovascular disease, cerebrovascular disease, arrhythmia, and rheumatologic disease were considered variables that could potentially contribute to fracture risk either through direct bone modeling or altering patients' propensity to fall [15-17]. Additionally, age, sex, race, body mass index (BMI), duration of dialysis prior to transplant, type of transplant (living vs. deceased), panel reactive antibody titers, and episodes of rejection were recorded as patient characteristics at time of transplant that could potentially serve as confounders. 
Medication use, namely types of chronic immunosuppression (mycophenolate mofetil, mycophenolic acid, tacrolimus, cyclosporine, azathioprine, sirolimus or everolimus, and corticosteroids), was identified by use in the year prior to index as found in Medicare Part B and D data.

\section{Statistical analysis}

We used unadjusted and multivariable-adjusted, conditional logistic regression to examine the association of case (hip fracture) vs. control status and prior statin use in first time KTRs. The estimate of association was expressed as odds ratios (OR) and corresponding 95\% confidence intervals $(\mathrm{CI})$. Odds ratios were calculated for any vs. non-use as well as for lesser or higher use, respectively, vs. non-use. All of the baseline characteristics and comorbidities detailed in Table 1 (except the factors used for the matching of controls) were included in the multivariate analysis.

Since the dataset was expected to contain incomplete data for some variables, we assumed the data to be missing at random and used multiple imputation to create and analyze 40 imputed datasets. [18] Methodologists currently regard multiple imputation as a state-of-theart technique because it improves accuracy and statistical power relative to other missing data techniques. Incomplete variables were imputed under fully conditional specification and the imputation model included all the variables in the analysis model plus a fixed effect for pair id to account for the matching of controls to cases $[19,20]$. Model parameters were estimated applying the analysis model to each imputed data set separately. These estimates and their standard errors were combined using Rubin's rules.

\section{Results}

\section{Patient selection and baseline characteristics}

We identified 231 cases of hip fractures amongst first time KTRs that fulfilled all stated inclusion and exclusion criteria between 2007 and 2011. We then selected 15,575 control KTRs matched on age, sex, race, and time to transplant. The number of matched KTR controls per case ranged between 1 and 225, with a median of 56 (interquartile range: 29; 93). The mean difference in the matching factors age and time since transplant between cases and controls was 1.72 years and 0.3 years, respectively (Additional file 1: Table S2). These results reflect matching well within the bounds allowed (3 years for age and 1 year for time since transplant). Cases had a higher prevalence of diabetes mellitus, cardiovascular disease, cerebrovascular disease, arrhythmia, and rheumatologic disease. Additionally, cases had higher use of corticosteroids, mTor inhibitors, cyclosporine, and azathioprine (Table 1). In the year prior to index date,
$64.1 \%$ of cases and $60.3 \%$ of controls exhibited any prior statin use. Additionally, $37.2 \%$ of cases and $33.9 \%$ of controls were denoted as adherent, having had greater than $80 \%$ of proportion of days covered (statin prescriptions covering at least 292 out of 365 days preceding the index date) (Table 2).

The percentage of missing values across the 4 variables incompletely recorded ranged from $<0.1 \%$ (history of acute rejection) to $14.8 \%$ (panel-reactive antibody titer). In total 3549 out of 15,806 records (22\%) were incomplete. More cases had PRA missing compared to controls (23\% vs. $15 \%)$. When performing a complete case analysis of 12,257 patients with all information, an additional 70 case-control groups (representing 2687 patients) were dropped because of no variability in the exposure variable (all statin users or all non-users) within the case-control group. This would leave an analysis sample of only 9570 patients, for a loss of $40 \%$ of the originally identified patients, thus further substantiating the use of multiple imputation.

\section{Associations between statin use and fracture}

The unadjusted conditional odds ratio of hip fracture (vs. control) status with any vs. no statin use was 1.17 $(0.89-1.54)$, and those with lesser $(<80 \%$ of PDC), and higher (>80\% PDC) statin use were $1.16(0.83-1.62)$, and $1.18(0.86-1.61)$, respectively. The multivariable adjusted odds ratios pointed into the opposite direction, but remained non-significant: 0.89 (0.67-1.19), 0.93 (0.66$1.31)$, and $0.87(0.63-1.20)$ for any, lesser, and higher use, respectively (Table 2).

\section{Discussion}

Using a nation-wide registry for KTRs, we found no clear association between the occurrence of a hip fracture event and prior use of statins. We undertook this study based on the findings of experimental research supporting the beneficial effects of statin on bone health and on clinical evidence from observational studies in the general and other subpopulations that supported such putative benefits. We capitalized on the availability of prescription drug information from Medicare Part D, which now permits large-scale studies of medication effectiveness in transplant and other Medicare insured populations. Considering the elevated hip fracture risk in KTRs, any modification of this vulnerability that can be achieved through better understanding of medication effects appears worthwhile.

The mechanisms by which statins can theoretically affect bone health are multifactorial. They have been shown to increase expression of bone morphogenetic protein-2 (BMP-2), resulting in increased osteoblast differentiation and consequent mineralization [21]. Statins 
Table 1 Characteristics of hip fracture cases and matched controls

\begin{tabular}{|c|c|c|c|c|c|}
\hline \multirow[t]{2}{*}{ Variable } & \multicolumn{2}{|l|}{ Cases $(n=231)$} & \multicolumn{2}{|c|}{ Controls $(n=15,575)$} & \multirow[t]{2}{*}{$P$-value } \\
\hline & Mean or $n$ & $\pm \mathrm{SD}$ or $\%$ & Mean or $n$ & $\pm \mathrm{SD}$ or $\%$ & \\
\hline \multicolumn{6}{|l|}{ Matched } \\
\hline Age (years) ( \pm 3 years) & 51.8 & \pm 12.9 & 51.2 & \pm 10.4 & 0.09 \\
\hline Median (IQR) & $54(42-61)$ & & $53(44-58)$ & & \\
\hline Male & 108 & 46.7 & 9308 & 59.8 & - \\
\hline African American & 26 & 11.3 & 1126 & 7.2 & - \\
\hline Time since transplant ( \pm 1 year) & 6.9 & \pm 5.3 & 4.6 & \pm 4.0 & 0.70 \\
\hline Median (IQR) & $6.1(2.6-10.0)$ & & $3.4(1.3-7.2)$ & & \\
\hline \multicolumn{6}{|l|}{ Not Matched } \\
\hline Hispanic ethnicity & 42 & 19.0 & 5143 & 33.4 & $<0.01$ \\
\hline Missing & 10 & 4.3 & 184 & 1.2 & \\
\hline Body mass index $\left(\mathrm{kg} / \mathrm{m}^{2}\right)$ & 26.0 & \pm 5.0 & 27.3 & \pm 5.2 & 0.01 \\
\hline Median (IQR) & $25.0(22.2-29.3)$ & & $26.8(23.5-30.7)$ & & \\
\hline Missing & 31 & 13.4 & 1417 & 9.1 & \\
\hline Time since ESRD (years) & 9.9 & \pm 5.2 & 8.1 & \pm 4.4 & 0.12 \\
\hline Median (IQR) & $9.3(5.7-12.9)$ & & $7.4(4.8-10.6)$ & & \\
\hline \multicolumn{6}{|l|}{ Comorbidities, recorded history of } \\
\hline Diabetes mellitus & 195 & 84.4 & 11,594 & 74.4 & $<0.01$ \\
\hline Cardiovascular disease & 204 & 88.3 & 11,694 & 75.1 & $<0.01$ \\
\hline Cerebrovascular disease & 101 & 43.7 & 3988 & 25.6 & $<0.01$ \\
\hline Arrhythmia & 82 & 35.5 & 4205 & 27.0 & 0.14 \\
\hline Rheumatologic disease & 30 & 13.0 & 1382 & 8.9 & 0.32 \\
\hline \multicolumn{6}{|l|}{ Transplant-related } \\
\hline Living (vs. deceased) donor & 56 & 24.2 & 3824 & 24.6 & 0.79 \\
\hline Acute rejection, history of & 32 & 13.9 & 1871 & 12.0 & 0.72 \\
\hline Missing & 0 & 0.0 & 22 & 0.1 & \\
\hline PRA $>80 \%$ & 14 & 7.9 & 824 & 6.2 & 0.59 \\
\hline Missing & 53 & 22.9 & 2285 & 14.7 & \\
\hline \multicolumn{6}{|l|}{ Immunosuppressant drugs } \\
\hline Tacrolimus & 125 & 54.1 & 9807 & 63.0 & 0.64 \\
\hline Cyclosporine & 71 & 30.7 & 2861 & 18.4 & $<0.01$ \\
\hline MMF/mycophenolic acid & 153 & 66.2 & 11,138 & 71.5 & 0.20 \\
\hline Azathioprine & 29 & 12.6 & 551 & 3.5 & $<0.01$ \\
\hline mTor inhibitor & 37 & 16.0 & 1536 & 9.9 & $<0.01$ \\
\hline Corticosteroid & 187 & 81.0 & 10,681 & 68.6 & $<0.01$ \\
\hline Bisphosphonate use & 61 & 26.4 & 2111 & 13.6 & $<0.01$ \\
\hline
\end{tabular}

ESRD end-stage renal disease; MMF mycophenolate mofetil; PRA panel-reactive antibodies

\$Obtained from a univariate conditional logistic regression model using a complete-case analysis. A $p$-value for male sex and African American race cannot be computed as these variables were hard matched

may also alter the mevalonate pathway to inhibit protein prenylation and subsequently decrease osteoclast resorption [4]. In conjunction with the known antiinflammatory effect, these cellular modifications provide a biological plausibility for hip fracture risk attenuation. Several studies support the association between statin use and increased trabecular bone volume [5]. However, the doses utilized in these animal studies, 5 or $10 \mathrm{mg} /$ $\mathrm{kg} /$ day of simvastatin, were considerably higher than what is used in clinical practice and may partially explain the inconclusive evidence from observational studies in humans [5]. 
Table 2 Statin use in hip fracture cases and their controls and measures of association

\begin{tabular}{|c|c|c|c|c|c|c|}
\hline \multirow[t]{2}{*}{ Statin Use Exposure } & \multicolumn{2}{|c|}{$\begin{array}{l}\text { Cases } \\
(n=231) \\
\end{array}$} & \multicolumn{2}{|c|}{$\begin{array}{l}\text { Controls } \\
(n=15,575) \\
\end{array}$} & \multicolumn{2}{|c|}{$\begin{array}{l}\text { Odds Ratios } \\
\text { (95\% Confidence Intervals) }\end{array}$} \\
\hline & $n$ & $\%$ & $n$ & $\%$ & Unadjusted & Adjusted \\
\hline No Use & 83 & 35.9 & 6181 & 39.7 & 1.0 (referent) & 1.0 (referent) \\
\hline Any Use & 148 & 64.1 & 9394 & 60.3 & $1.17(0.89-1.54)$ & $0.89(0.67-1.19)$ \\
\hline Lesser Use (PDC < 80\%) & 62 & 26.8 & 4111 & 26.4 & $1.16(0.83-1.62)$ & $0.93(0.66-1.31)$ \\
\hline Higher Use (PDC >80\%) & 86 & 37.2 & 5283 & 33.9 & $1.18(0.86-1.61)$ & $0.87(0.63-1.20)$ \\
\hline
\end{tabular}

Note: Separate models were fit to study i) any statin use, or ii) lesser and higher statin use compared with no statin use

From conditional logistic regression models of cases and control sets matched on age ( \pm 3 years), sex, race, and time since kidney transplant ( \pm 1 year). Adjusted models controlled for Hispanic ethnicity, body mass index, time since incident end-stage renal disease, comorbidities (diabetes, cardiovascular disease, cerebrovascular disease, arrhythmia, rheumatologic disease), transplant related factors (living vs. deceased donor, history of acute rejection, maximum panel-reactive antibody titer), and individual immunosuppressant drugs used in the year prior to the index date (tacrolimus, cyclosporine, mycophenolate mofetil/mycophenolic acid, azathioprine, sirolimus/everolimus, corticosteroids)

Previous studies on this topic in the general population have yielded conflicting data. In a study of U.S. Veterans, mostly men, Scranton et al. concluded that patients had a $36 \%$ reduced fracture risk if they were prescribed a statin more than once [8]. A population-based, matched case-control study from a large integrated health care organization in Southern California also found a beneficial association between hip fracture status and statin use [adjusted OR $0.68(0.62-0.74)$ ] [22]. The large-scale Finnish study conducted by HelinSalmivaara et al. also described favorable outcomes for statin users amongst post-menopausal women age 5080 [7]. Those patients that reported having used statins for at least 5 years had reduced hip fractures rates compared to controls adherent to hypertension drugs [HR $0.71(0.58-0.86)]$ and a randomly selected cohort of the Finnish registry [HR $0.67(0.55-0.87)]$. Of note, there was no association between degree of statin adherence and hip fracture risk. Other observational studies of either cohort or case-control design did not detect any associations. The prospective Women's Health Initiative study did not identify an association between statin use and fracture risk in 93,716 postmenopausal women [HR $1.22(0.83-1.81)$ ] [23]. Similarly, van Staa et al. found no association of hip fracture status with prior statin exposure in the large and generalizable United Kingdom General Practice Research Database [OR 0.59 (0.31-1.13)] [24]. Lastly, a Danish national cohort study conducted by Hansen et al. determined an elevated risk of first time fracture within a kidney transplant cohort versus the general background population in multivariate analysis [HR 1.82 (1.62-2.06)]. Lipid-modifying drugs were not associated with fracture risk in the kidney transplant sub-cohort [HR 1.16 (0.87-1.56)]. However, of the 265 observed fractures among KTR only 10\% ( 27) were hip fractures [25]. Distinguishing type of fracture may be relevant since a recent study found a protective association between statin use and hip fracture, but not with the outcomes of all fractures, lower-extremity fractures, or upper-extremity fractures [26].
While ample information on the efficacy of statins on reducing cardiovascular risk is available, there is limited information from randomized trials regarding their putative effect on fracture rates. Post-hoc analysis of earlier randomized cardiovascular trials had demonstrated a lack of reduced fracture risk [27], but the Justification for the Use of statins in Prevention: an Intervention Trial Evaluating Rosuvastatin (JUPITER) trial was the first a priori examination of this hypothesis. This double-blind placebo-controlled study produced no evidence on the efficacy of high-dose rosuvastatin use for the reduction of fracture risk [HR $1.06(0.88-1.28)$ ] [28]. However, this trial focused on a rather healthy population that was at much lower fracture risk compared to patients with advanced kidney disease including those with a functioning kidney transplant.

Indeed, bone pathology in kidney disease is quite different from the bone disease experienced by the general population. Uremia, metabolic acidosis, an abnormal vitamin D-parathyroid hormone-FGF-23 axis, and an inflammatory milieu are all factors that contribute to a significantly higher risk of fracture in those with kidney disease. While mitigated after transplant, where some of the physiological processes controlling bone metabolism and health may be reinstated, the effect of prior kidney disease and the long-term use of corticosteroids and other immunosuppressive medications produce a distinct bone disease and render these patients at longterm elevated fracture risk. Thus, for bone disease, evidence garnered from the general population may not be applicable to the kidney transplant population.

There are important differences between the interactions observed with cyclosporine versus tacrolimus and the statins. Co-administration of statin with cyclosporine results in a 6-15 fold increase in statin plasma levels while a number of pharmacological studies have demonstrated relatively little effect of tacrolimus coadministration on statin levels [29-31]. Traditionally, the CNI-statin interaction has been thought to center on competitive inhibition of the CYP3A4 enzyme. However, 
the important differences observed with cyclosporine versus tacrolimus co-administration on statin plasma levels may be a result of their differential effects on other enzymes important for statin transport and metabolism such as OATP1B1 [32]. In any case, an abundance of caution likely exists around calcineurin inhibitor and statin co-administration. Many of the more potent statins that are also CYP3A4 inhibitors are less frequently employed. Consequently, the alternatively metabolized options, pravastatin and fluvastatin, which are less potent HMG-CoA reductase inhibitors are more commonly prescribed in KTRs. Rosuvastatin, a much more potent non-CYP3A4 inhibiting statin, may be less commonly prescribed amongst KTRs given concerns regarding the development of proteinuria and its higher price [33]. Thus, statins may be relatively 'underdosed' in tacrolimus co-treated patients. Unfortunately, our study is to small to support meaningful tests for effect modification or differences in association within class.

In the general population, bone mineral density (BMD) and the fracture risk assessment tool (FRAX) serve as additional calculations to further stratify risk. Due to the aforementioned complex and distinct bone disease in KTRs, the accuracy of these tools is uncertain. The pathology associated with kidney disease is not limited to generalized density and observational studies regarding BMD assessed by dual energy $\mathrm{x}$-ray absorptiometry produce conflicting results [34, 35]. FRAX scores have not been universally accepted for kidney transplants, but an isolated study by Naylor et al. reported predictions for 10-year risk based on FRAX comparable to observed risk [36]. As the investigators noted, a cohort study of 458 patients over a mean of 6 years is not definitive and requires independent validation with larger cohorts. In our study, limited look back windows for the ascertainment of fractures in the more distant past limit the ability to incorporate FRAX into our analysis.

While accounted for in multivariate analysis, cardiovascular disease, diabetes mellitus, cerebrovascular disease, arrhythmias, rheumatologic disease, and steroid use were all more prevalent in cases. Indeed, the direction of the associations swung the opposite way (albeit all without reaching significance) after adjustment for these characteristics, which may motivate speculation that accounting for any residual, unobserved confounding may further push the associations towards statin protection. Clearly, despite being the largest study on the topic to date, at least to our knowledge, similar analyses in larger individual or pooled databases are warranted to achieve further precision on the association of interest.

Other limitations of our study relate to the relatively limited look back window for statin exposure (and for the covariates on immunosuppressant use). This is owing to the relatively recent introduction of prescription drug coverage in Medicare insured patients via Part D. While we could conduct the same study with the requirement of longer prescription drug coverage prior to the index date (e.g., 2 or 3 years), and have attempted to do so, sample sizes would diminish considerably and insufficient numbers of cases would be identified to support adequate multivariable adjustment, thus inducing less precision and increased likelihood of bias. Therefore, we acknowledge not having captured the full duration of statin exposure and being limited to 1 year of statin use ascertainment. Given the possibility that the mechanism of bone protection may require longer periods of statin exposure, our study could have potentially missed a protective effect. Finally, this study was conducted in U.S. KTR with Medicare coverage; its findings may not generalize to KTR with other insurance coverage or in other countries or health care systems.

\section{Conclusions}

In conclusion, this large population-based study of firsttime KTRs did not identify an independent association of hip fracture events with any or adherent statin use. These findings need to be interpreted in light of the observational nature of the study as well as the relatively limited number of hip fractures identified, which renders limited power to identify smaller, but clinically meaningful associations.

\section{Additional file}

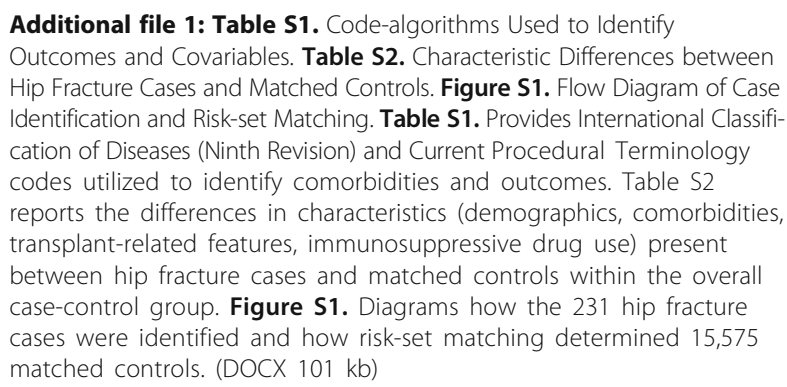

\section{Abbreviations}

CNI: Calcineurin inhibitor; ESRD: End-stage renal disease; KTR: Kidney transplant recipients; PDC: Proportion of days covered; USRDS: United States Renal Data System

\section{Acknowledgements}

We wish to thank Dr. and Mrs. Harold Selzman for their generous gift that supported this project.

\section{Funding}

CV was supported by a gift from Dr. and Mrs. Harold Selzman. CRL receives grant support through a Mentored Clinical and Population Research Award from the American Heart Association (Western State Affiliate) and a Norman S. Coplon Extramural Grant for Clinical Applied Research from Satellite

Healthcare. SSN was funded by a fellowship grant from Sanofi-Aventis. The Stanford nephrology fellowship program was supported by grant

T32DK007357 from the National Institutes of Health. WCW receives support through the endowed Gordon A. Cain Chair in Nephrology at Baylor College of Medicine. 


\section{Availability of data and materials}

All relevant data are drawn from United States Renal Data System and are included within paper.

\section{Authors' contributions}

CV drafted manuscript, performed bibliographic search, and interpreted results of analysis. WCW was instrumental to conception, drafting study design, and interpreting analysis. CRL substantially influenced conception and study design; CRL also provided significant contributions to interpretation of results. MMR participated in study design, determined and performed statistical analysis, and made tables. SSN was involved in interpretation of results. SDN and VR made important contributions to revising manuscript for important intellectual content. Each author contributed important intellectual content to manuscript drafting and revision. All authors approved the final manuscript.

\section{Competing interests}

Dr. Winkelmayer reports having served as an advisor or consultant, unrelated to the topic of this manuscript, to Akebia, AMAG, Amgen, Astra-Zeneca, Medtronic, Relypsa, Vifor-Fresenius Medical Care Renal Pharma, and Zoll. The authors declare that they have no competing interests.

\section{Consent for publication}

Not Applicable.

\section{Ethics approval and consent to participate}

This study is in accordance with Helsinki Declaration. This study was approved by institutional review boards at Stanford University (IRB-17904) and Baylor College of Medicine ( $\mathrm{H}-36408)$. The study was granted a waiver of informed consent.

Work was conducted under a data use agreement between WCW and the National Institute of Diabetes, Digestive and Kidney Diseases (NIDDK). This manuscript was reviewed and approved for publication by an officer of the National Institute of Diabetes and Digestive and Kidney Diseases. Data reported herein were supplied by the US Renal Data System. Interpretation and reporting of these data are the responsibility of the authors and in no way should be seen as official policy or interpretation of the US Government.

\section{Publisher's Note}

Springer Nature remains neutral with regard to jurisdictional claims in published maps and institutional affiliations.

\section{Author details}

'Baylor College of Medicine, Section of Nephrology, One Baylor Plaza, BCM 620 - 11D32.5, Houston, TX 77030, USA. '2Division of Nephrology, Stanford University School of Medicine, 777 Welch Road Suite DE, Palo Alto, CA 94304, USA. ${ }^{3}$ Division of Nephrology, Department of Medicine, Stanford University School of Medicine, 1070 Arastradero Road \# 3C11C, Palo Alto, CA 94304, USA. ${ }^{4}$ Mayo Clinic Arizona, 5777E Mayo Blvd, Phoenix, AZ 85012, USA. ${ }^{5}$ Baylor College of Medicine, Section of Nephrology, One Baylor Plaza, Ste 100-37D, Houston, TX 77030, USA. ${ }^{6}$ Section of Nephrology, Michael E. DeBakey VA Medical Center, 2002 Holcombe Boulevard, \#111-J, Houston, TX 77030, USA. 'Baylor College of Medicine, Section of Nephrology, One Baylor Plaza, BCM 395, Houston, TX 77030, USA.

Received: 8 November 2016 Accepted: 19 April 2017 Published online: 01 May 2017

\section{References}

1. Brauer CA, Coca-Perraillon M, Cutler DM, Rosen AB. Incidence and mortality of hip fractures in the United States. JAMA. 2009;302:1573-9.

2. Alem AM, Sherrard DJ, Gillen DL, Weiss NS, Beresford SA, Heckbert SR, Wong C, Stehman-Breen C. Increased risk of hip fracture among patients with end-stage renal disease. Kidney international. 2000;58:396-9.

3. Ball AM, Gillen DL, Sherrard D, Weiss NS, Emerson SS, Seliger SL, Kestenbaum BR, Stehman-Breen C. Risk of hip fracture among dialysis and renal transplant recipients. JAMA. 2002;288:3014-8.

4. Yamashita M, Otsuka F, Mukai T, Yamanaka R, Otani H, Matsumoto Y, Nakamura E, Takano M, Sada KE, Makino H. Simvastatin inhibits osteoclast differentiation induced by bone morphogenetic protein-2 and RANKL through regulating MAPK, AKT and Src signaling. Regulatory peptides. 2010; 162:99-108.

5. Mundy G, Garrett R, Harris S, Chan J, Chen D, Rossini G, Boyce B, Zhao M, Gutierrez G. Stimulation of bone formation in vitro and in rodents by statins. Science (New York, NY). 1999;286:1946-9.

6. Wang PS, Solomon DH, Mogun H, Avorn J. HMG-CoA reductase inhibitors and the risk of hip fractures in elderly patients. JAMA. 2000;283:3211-6.

7. Helin-Salmivaara A, Korhonen MJ, Lehenkari P, Junnila SY, Neuvonen PJ, Ruokoniemi P, Huupponen R. Statins and hip fracture prevention-a population based cohort study in women. PloS one. 2012;7:e48095. doi:10. 1371/journal.pone.0048095.

8. Scranton RE, Young M, Lawler E, Solomon D, Gagnon D, Gaziano JM. Statin use and fracture risk: study of a US veterans population. Archives of internal medicine. 2005;165:2007-12.

9. Kidney Disease Improving Global Outcomes (KDIGO) Lipid Work Group. KDIGO Clinical Practice Guideline for Lipid Management in Chronic Kidney Disease. Kidney Int, Suppl 2013;3:259-305.

10. Pilmore HL, Skeans MA, Snyder JJ, Israni AK, Kasiske BL. Cardiovascular disease medications after renal transplantation: results from the Patient Outcomes in Renal Transplantation study. Transplantation. 2011;91:542-51.

11. Gaston RS, Kasiske BL, Fieberg AM, Leduc R, Cosio FC, Gourishankar S, Halloran P, Hunsicker L, Rush D, Matas AJ. Use of cardioprotective medications in kidney transplant recipients. American journal of transplantation : official journal of the American Society of Transplantation and the American Society of Transplant Surgeons. 2009;9:1811-5.

12. Palmer SC, Navaneethan SD, Craig JC, Perkovic V, Johnson DW, Nigwekar SU, Hegbrant J, Strippoli GF. HMG CoA reductase inhibitors (statins) for kidney transplant recipients. The Cochrane database of systematic reviews. 2014. doi:10.1002/14651858.

13. Sukumaran Nair S, Lenihan CR, Montez-Rath ME, Lowenberg DW, Chertow GM, Winkelmayer WC. Temporal trends in the incidence, treatment and outcomes of hip fracture after first kidney transplantation in the United States. Am J Transplant. 2014;14:943-51.

14. Saran RLY, Robinson B, et al. US Renal Data System 2015 Annual Data Report: Epidemiology of Kidney Disease in the United States. American journal of kidney diseases : the official journal of the National Kidney Foundation. 2016;67:S1-S434.

15. Sennerby U, Melhus H, Gedeborg R, Byberg L, Garmo H, Ahlbom A, Pedersen NL, Michaelsson K. Cardiovascular diseases and risk of hip fracture. JAMA. 2009;302:1666-73.

16. Fan Y, Wei F, Lang Y, Liu Y. Diabetes mellitus and risk of hip fractures: a meta-analysis. Osteoporosis international : a journal established as result of cooperation between the European Foundation for Osteoporosis and the National Osteoporosis Foundation of the USA. 2016;27:219-28.

17. van Staa TP, Geusens P, Bijlsma JW, Leufkens HG, Cooper C. Clinical assessment of the long-term risk of fracture in patients with rheumatoid arthritis. Arthritis and rheumatism. 2006;54:3104-12.

18. Rubin DB: Multiple imputation for nonresponse in surveys., vol. 81: John Wiley \& Sons; 2004.

19. Montez-Rath ME, Winkelmayer WC, Desai M. Addressing missing data in clinical studies of kidney diseases. Clin J Am Soc Nephrol. 2014;9:1328-35.

20. Van Buuren S, Brand JPL, Groodhuis-Oudshoorn CGM, Rubin DB. Fully conditional specification in multivariate imputation. J Stat Comp Simul. 2006;76:1049-64.

21. Alam S, Ueki K, Nakagawa K, Marukawa K, Hashiba Y, Yamamoto E, Sakulsak N, Iseki S. Statin-induced bone morphogenetic protein (BMP) 2 expression during bone regeneration: an immunohistochemical study. Oral surgery, oral medicine, oral pathology, oral radiology, and endodontics. 2009;107:22-9.

22. Adams AL, Shi JM, Reynolds K, Haque R, Cheetham TC, Kawatkar AA, Fithian DC, Jacobsen SJ. Statins and hip fracture risk in men: a population-based case-control study. Annals of epidemiology. 2015;25:844-8.

23. LaCroix AZ, Cauley JA, Pettinger M, Hsia J, Bauer DC, McGowan J, Chen Z, Lewis CE, McNeeley SG, Passaro MD, et al. Statin use, clinical fracture, and bone density in postmenopausal women: results from the Women's Health Initiative Observational Study. Annals of internal medicine. 2003:139:97-104.

24. van Staa TP, Wegman S, de Vries F, Leufkens B, Cooper C. Use of statins and risk of fractures. Jama. 2001;285:1850-5.

25. Hansen D, Olesen JB, Gislason GH, Abrahamsen B, Hommel K. Risk of fracture in adults on renal replacement therapy: a Danish national cohort study. Nephrol Dial Transplant. 2016;31:1654-62. 
26. Ward IM, Mortensen EM, Battafarano DF, Frei CR, Mansi I. Association of statins and risk of fractures in a military health system: a propensity scorematched analysis. The Annals of pharmacotherapy. 2014;48:1406-14.

27. Reid IR, Haque W, Emberson J, Baker J, Tonkin A, Hunt D, MacMahon S, Sharpe N. Effect of pravastatin on frequency of fracture in the LIPID study: secondary analysis of a randomised controlled trial. Long-term Intervention with Pravastatin in Ischaemic Disease. Lancet (London, England). 2001;357: 509-12.

28. Pena JM, Aspberg S, MacFadyen J, Glynn RJ, Solomon DH, Ridker PM. Statin therapy and risk of fracture: results from the JUPITER randomized clinical trial. JAMA internal medicine. 2015;175:171-7.

29. Lemahieu WP, Hermann M, Asberg A, Verbeke K, Holdaas H, Vanrenterghem $Y$, Maes BD. Combined therapy with atorvastatin and calcineurin inhibitors: no interactions with tacrolimus. American journal of transplantation : official journal of the American Society of Transplantation and the American Society of Transplant Surgeons. 2005;5:2236-43.

30. Asberg A, Hartmann A, Fjeldsa E, Bergan S, Holdaas H. Bilateral pharmacokinetic interaction between cyclosporine $\mathrm{A}$ and atorvastatin in renal transplant recipients. American journal of transplantation : officia journal of the American Society of Transplantation and the American Society of Transplant Surgeons. 2001;1:382-6.

31. Amundsen R, Christensen H, Zabihyan B, Asberg A. Cyclosporine A, but not tacrolimus, shows relevant inhibition of organic anion-transporting protein 1B1-mediated transport of atorvastatin. Drug metabolism and disposition: the biological fate of chemicals. 2010;38:1499-504.

32. Romaine SP, Bailey KM, Hall AS, Balmforth AJ. The influence of SLCO1B1 (OATP1B1) gene polymorphisms on response to statin therapy. The pharmacogenomics journal. 2010:10:1-11.

33. Alsheikh-Ali AA, Ambrose MS, Kuvin JT, Karas RH. The safety of rosuvastatin as used in common clinical practice: a postmarketing analysis. Circulation. 2005;111:3051-7.

34. Grotz WH, Mundinger FA, Gugel B, Exner V, Kirste G, Schollmeyer PJ. Bone fracture and osteodensitometry with dual energy $\mathrm{X}$-ray absorptiometry in kidney transplant recipients. Transplantation. 1994;58:912-5.

35. Akaberi S, Simonsen O, Lindergard B, Nyberg G. Can DXA predict fractures in renal transplant patients? American journal of transplantation : official journal of the American Society of Transplantation and the American Society of Transplant Surgeons. 2008;8:2647-51.

36. Naylor KL, Leslie WD, Hodsman AB, Rush DN, Garg AX. FRAX predicts fracture risk in kidney transplant recipients. Transplantation. 2014;97:940-5.

\section{Submit your next manuscript to BioMed Central and we will help you at every step:}

- We accept pre-submission inquiries

- Our selector tool helps you to find the most relevant journal

- We provide round the clock customer support

- Convenient online submission

- Thorough peer review

- Inclusion in PubMed and all major indexing services

- Maximum visibility for your research

Submit your manuscript at www.biomedcentral.com/submit

) Biomed Central 